\title{
2. SPECTROGRAPHIC OBSERVATIONS OF VISUAL BINARIES
}

\author{
R. M. Petrie and A. H. Batten \\ INTRODUCTION
}

A programme of spectroscopic observations of visual binaries has been followed now for some years at the Dominion Astrophysical Observatory. The aim has been to accumulate radial velocities, spectral types, and absolute magnitudes for a large number of systems so as to provide data for studies of physical characteristics of visual binaries. The work is far from complete and, indeed, can continue indefinitely: this paper gives an account of the results obtained at the present time.

The systems placed on the programme were selected according to the following criteria:

I. The separation of the two components is greater than 3 seconds of arc.

2. The fainter component is not fainter than ninth magnitude.

3. The system is located north of the celestial equator.

These criteria are required by the limitations of the instruments available at Victoria. Over 700 systems satisfying these criteria were extracted from Aitken's General Catalogue of Doubel Stars. In this paper we confine our attention to some 300 of these systems, for which there are sufficient data to justify the assumption that these are real physical systems. We have considered a system to be physical if one or more of the following criteria are met:

I. An astrometric orbit has been determined, or, at least, the relative motion of the pair is clearly the result of their orbital motion.

2. The component stars have common proper motion.

3. The radial velocities of the two stars differ by less than $10 \mathrm{~km} / \mathrm{sec}$. The sources of the data used in examining systems for these criteria were for I, Aitken's General Catalogue of Double Stars, for 2, the same Catalogue and Luyten (I), for 3, wherever possible our own (unpublished) determinations were used. For many systems, however, it is necessary to rely on the velocities given in Wilson's General Catalogue of Radial Velocities. The limit of ro km/ sec for the radial velocity difference has been rather arbitrarily chosen; it is an attempt to allow for the errors of measurement of the velocities of early-type components and also to allow for the line-of-sight component of any possible orbital motion. It is probable that the limit is wide enough to admit some systems into consideration which are really optical. However, of those systems for which both radial-velocity and proper-motion data are available, only two that would have been excluded by their proper motions are included because of their radial velocities. There is no system with common proper motion that would have been excluded from this list by its observed radial velocities.

\section{FREQUENCY OF MULTIPLE SYSTEMS}

The data described in the previous section may be used to estimate the proportion of binary systems which are in fact triple or even more complex. This has been done by counting the number of systems containing at least one component whose velocities are known or suspected to vary. We assume that the variation in the velocities of these components arises from orbital motions of a close pair of stars which is additional to the known orbital motion of the recognized wide pair. In the majority of cases this assumption is known to be correct, because of the character of the motion.

Our sample of 307 visual binary systems contains $9 \mathrm{r}$ with at least one component which shows, or is believed to show variable radial velocity. Of these $9 \mathrm{I}$, there are $\mathrm{r} 9$ systems of which both members are considered to vary in velocity. These numbers give an underestimate of the 
proportion of complex systems, because velocity data are not available for all 307 systems. There are 234 systems for which radial velocities of both components are known, and of these 82 , or 35 per cent, contain at least one component with variable velocity. Approximately 23 per cent of the 'triple' systems appear to be even more complex-these are the systems in which both members of the visual pair appear to have variable velocities.

Many of the $9 \mathrm{r}$ components considered to have variable velocity are known to be spectroscopic binaries and orbits have been determined for them. In other cases the range of variation is known or double lines have been observed in the spectrum. Thus it is very likely that most of the stars counted as varying in velocity are indeed members of spectroscopic binaries. Of course, some of the stars whose velocities are now thought to be variable will, upon further observation, prove to have constant velocities. On the other hand, some of those thought to have constant velocities will, in the same way, be found to be variable. Furthermore, a number of genuine triple systems will be excluded from consideration by our third criterion. When only a few observations of the velocity of a spectroscopic binary have been made, their average is likely to differ from the true systemic motion. 'Thus the two components of a visual binary are likely to appear to have radial velocities differing by more than $10 \mathrm{~km} / \mathrm{sec}$ if one of them is a spectroscopic binary which has not been well observed.

This last statement can be illustrated by considering only those systems for which both radial velocities are determined, and which also satisfy at least one of our other two criteria for the physical nature of the system. There are 54 such systems in our sample, and 21 of them, or nearly 39 per cent are triple or multiple. This is greater than the percentage found in the larger and less completely observed sample of 234 systems. Thus our third criterion tends to discriminate against the recognition of triple systems rather than in their favour.

We believe, therefore, that our value for the percentage of triple systems has not been inflated by observational selection. Moreover some well-known multiple systems, such as Mizar, have not been included in the present programme although they meet our criteria. Finally we have taken no account of optical companions which accompany many visual binaries. Some of these, e.g. $\mu$ Bootis are known to be associated with the principal pair. Others are difficult to observe--especially for radial-velocity determinations. Many of them, however, must be associated with the principal pair. The total percentage of multiple systems, amongst binary systems, may well be over 40 per cent. Since the percentage of binary stars amongst all stars is believed to be of the order of $5^{\circ}$ per cent, it seems likely that it is more probable for a star to have companions than for it to be entirely alone. This is a fact of considerable significance for theories of star formation.

\section{EFFECTS OF MULTIPLICITY IN SPECTROSCOPIC BINARIES}

It is natural to consider whether spectroscopic binaries exhibit the same proportion of multiple systems among their number as seems to be evident among visual binaries. The normal method of detecting triplicity in a spectroscopic binary is to look for changes in the systemic velocity. It is normally assumed that these changes are produced by the orbital motion of the close pair about a third body. In certain circumstances, however, this assumption may be wrong and apparent changes in the other orbital elements of the close pair may provide the clue to the true nature of the system. In this section we will show how the presence of an unrecognized third body can affect the determination of all the orbital elements.

Typical known spectroscopic triples are Algol and $\lambda$ Tau. The ratio of the two orbital periods is 250 in Algol and 8 in $\lambda$ Tau. These systems represent extremes of what seems to be a pattern for spectroscopic triples. A close pair is attended by a relatively distant companion which is not, however, distant enough to be optically resolvable. It is likely that many triple systems exist with their longer periods between these two extremes. In such systems the longer period 
would not necessarily be long compared with the interval over which spectroscopic observations are made. The observed radial velocities of the system would, therefore, contain two components of variation-one from each orbit. The amplitude in the long-period orbit would be small, and the variation of the systemic velocity of the close pair would not be easily detectable, but if the resulting velocities are used to obtain the orbital elements of the close pair these elements will all be altered by the unrecognized motion about the third body. Orbits determined from observations obtained at different epochs will differ from each other in all their elements.

It is very easy to see qualitatively that orbits must be apparently distorted in this way if the long-period motion is not recognized and allowed for. It is not easy to derive a quantitative estimate of the observable variation of the orbital elements. Only for the semi-amplitude of the velocity of the close pair is it possible readily to derive an upper limit by the use of an artificial and extreme example. Suppose that observations made near the ascending node of the shortperiod orbit are always made when the close pair is also at the ascending node of the longperiod orbit. Similarly observations near the descending node of the short-period orbit are always made at the descending node of the long-period orbit. Then, if the two semi-amplitudes of velocity variation are $K_{1}$ and $K_{2}$ it is evident that the observed $K$ is given by

$$
K=K_{1}+K_{2} \text {. }
$$

It is readily seen that the opposite extreme situation gives an observed $K$ of

$$
K=K_{1}-K_{2}
$$

For example, in the system of Algol, $K_{1} \approx 44 \mathrm{~km} / \mathrm{sec}, K_{2} \approx 10 \mathrm{~km} / \mathrm{sec}$. We should expect the observed $K$ to fluctuate, therefore, between the limits $34 \mathrm{~km} / \mathrm{sec}$ and $54 \mathrm{~km} / \mathrm{sec}$ if no allowance is made for motion in the long-period orbit (Fig. $\mathrm{I}$ ). A variation in $K$ between the limits of $4 \mathrm{I} \mathrm{km} / \mathrm{sec}$ and $46 \mathrm{~km} / \mathrm{sec}$ has actually been observed (2).

Variations in $K$ have already been noted in other systems. They cannot be explained on any reasonable hypothesis involving only two-body motion. For instance, there is a variation of about $3 \mathrm{~km} / \mathrm{sec}$ in values of $K$ determined for AR Cas which is, statistically, barely significant. A larger variation is known in the system HD 208947. The system AR Cas also shows variations in the other orbital elements. Apparent variations in $K$ which are hard to explain are also known in $\delta$ Cap and `Peg. Each of these last two systems has shown apparent changes in $K$ of about $5 \mathrm{~km} / \mathrm{sec}$. The spectra of these systems show sharp lines and there is little doubt that these apparent changes are significant.

It is clear that $e$ and $\omega$ will be affected by any distortion of the velocity curve since their values depend on the shape of the curve. A similar artificial example was constructed of a triple system in which the short-period orbit had $e=0.40, \omega=0^{\circ}$ (Fig. 2). The long-period orbit was again assumed circular. It was found that an extreme variation between $e=0.35$ and $e=0.45$ might be encountered in a system in which the ratio of the periods was similar to that of Algol. It is of interest that spectroscopic determinations of the orbital eccentricity of Algol have ranged from virtually zero to 0.09 (2). McLaughlin ascribed the highest of these values to just the effect we are discussing. Photometrically, the eccentricity of Algol's shortperiod orbit is zero. It will be remembered that the disparity of photometric and spectroscopic determinations of the orbital eccentricity of many close binaries and the associated uncertainty in the longitude of periastron were considered of special significance by Struve. The disparity was particularly evident in systems whose orbits were photometrically circular, but whose radial velocities required an orbital eccentricity of up to $0^{\circ} I$ (3). The disparity was usually regarded by Struve as evidence of gas-streaming within the system although it is much smaller than the gross distortions observed in such systems as U Cep and RW Tau. It seems at least possible that many systems in which there is a difference between the orbital eccentricities obtained from photometric and spectroscopic observations are really unrecognized triple 
systems. The hypothesis could, in many instances, be tested by obtaining later epoch orbits. If all the orbital elements show small variations, it is very likely that a system is triple.

It should be pointed out that the conventional method of detecting a triple system may sometimes fail. This method is to look for and to analyse a real variation in the systemic velocity.

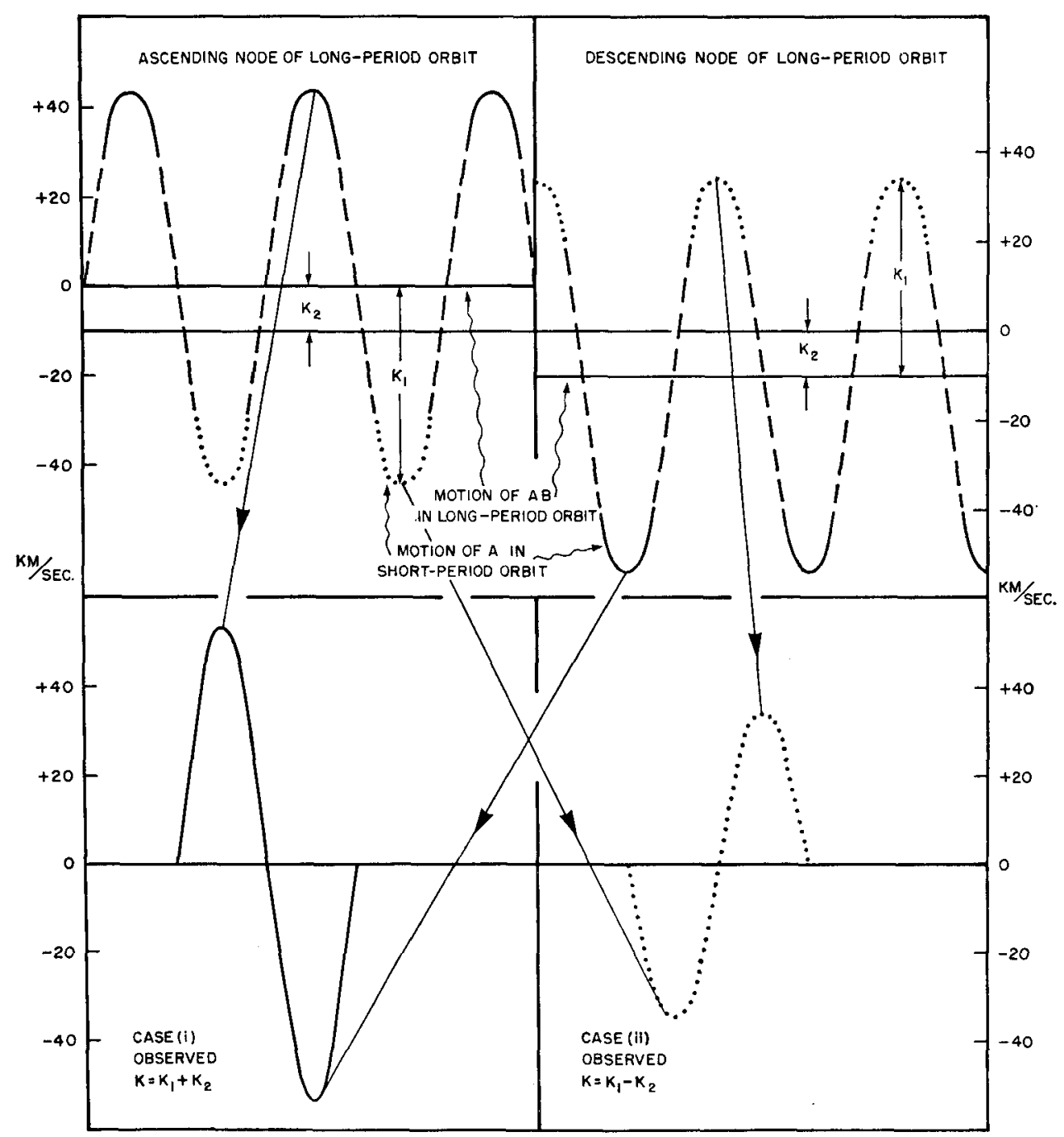

MAXIMUM EFFECT OF LONG-PERIOD MOTION IN ALGOL ON OBSERVED AMPLITUDE OF RADIAL-VELOCITY VARIATION

$\frac{K_{2}}{K_{1}} \approx \frac{1}{4 \cdot 4}: \frac{P_{2}}{P_{1}} \approx 250$

FIG. I

If all the orbital elements are in error, however, some of these errors may be reflected in the derived value of the systemic velocity. This is especially true when the short-period orbit has an appreciable eccentricity. For $e=0.4$ a difference of $\sim 5 \mathrm{~km} / \mathrm{sec}$ can be produced in the 
derived systemic velocity as determined at different relative phases of the two orbits-even though the true mean systemic velocity is the same for the different series of observations. Thus the variation of the apparent systemic velocity of a close pair in the triple system may not be

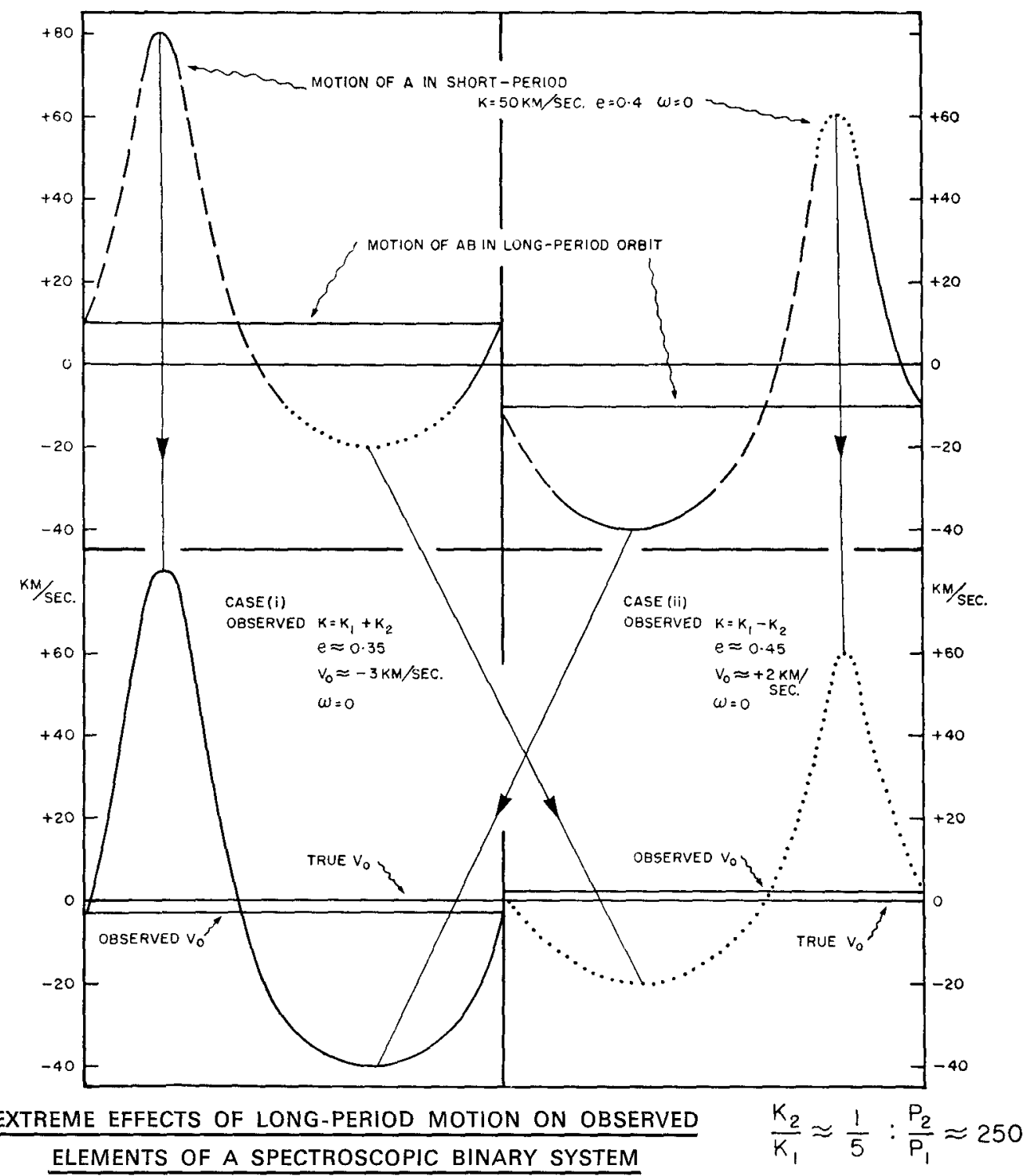

FIG. 2

the true motion of the close pair about the centre of mass of the whole system and may not even be periodic.

Observations of many spectroscopic binaries do not conform strictly to the predictions of two-body motion and their velocity curves cannot, therefore, be represented exactly by an 
equation for elliptic motion. The failure of photometric and spectroscopic elements to agree is almost commonplace, and we have also shown that a number of systems are known in which hitherto unexplained apparent changes in the orbital elements have been observed. It appears possible that these phenomena may often be explained by introducing the assumption that a third body exists in the system. Thus it appears possible that the proportion of triple (or multiple) systems amongst spectroscopic binaries is comparable with the $30-40$ per cent found for visual binaries, in the previous section.

\section{HERTSZPRUNG-RUSSELL DIAGRAM FOR WELL-OBSERVED PHYSICAL SYSTEMS}

We have made a Hertzsprung-Russell diagram on which are plotted all the systems in our sample which we considered physical and for which spectra of both components are available at Victoria. Each of us independently examined all the available spectra with an eyepiece and assigned spectral types on the basis of this examination. In nearly all cases our estimates were within two sub-types. In all the remaining cases it was possible for us to reach agreement after further examination. The spectral types thus assigned were used in plotting the HR diagram The absolute magnitudes were obtained in the following ways:

I. If both stars in a system were type $\mathrm{A}_{3}$ or earlier the absolute magnitude was obtained by measuring the equivalent width of $\mathrm{H} \gamma$. The revised calibration was used (4). Absolute magnitudes obtained with this calibration agree well with the photometric distance scale. No reference was made to observed values of the apparent magnitude difference, $\Delta m$, between the two components.

2. If one component of a system was earlier than $\mathrm{A}_{3}$, its absolute magnitude was deternined from the $\mathrm{H} \gamma$ intensity. The observed value of $\Delta m$ was used to place the other component in the HR diagram. Sources of $\Delta m$-values used were Muller (5), Wallenquist (6), Eggen (7) and Tolbert (8). A few systems with no reliable observed $\Delta m$ wete plotted by fitting the later component either to the main sequence or to the giant branch-according as their spectra indicated low or high luminosity.

3. If both components were later than $A_{3}$ the fainter star was assumed to lie on the main sequence and the observed value of $\Delta m$ was used to plot the position of the other star. This often resulted in both components being found on the main sequence. In one instance it seemed more appropriate to place the fainter component on the giant branch.

To fit the late-type components to the main sequence it was necessary to choose a standard (zero-age) main sequence. We used here the standard main sequence given by Arp (9). It differs slightly from the most modern versions given by Johnson (Io) and Blaauw (II) but the differences are too small to affect our conclusions-especially since they are confined to types later than B8. In fact Arp's sequence has been extended to the earliest types by using Blaauw's figures. The giant sequence plotted on the diagram is essentially that given by Keenan (I2).

The resulting HR diagram is shown in Fig. 3. Although we used spectral types to make up the diagram, the horizontal scale has been made linear in $B-V$, using Johnson's correlation between $B-V$ and spectral type (ro). The rapid contraction of the spectral type scale at colours bluer than $-0 \cdot 1$ should be kept in mind because it means that the usual classification errors for spectra earlier than B8 are very small on our plot.

The main features of the usual HR diagram are represented by these visual binaries, that is, most of the stars fall approximately along the main sequence and there is a very small population on the ordinary giant branch. There is, however, a tendency for the stars generally to be brighter than the unevolved main sequence and only a few stars are fainter. The average amount is at least 0.5 magnitude, and is not likely, in the mean, to be observational error. The great majority of the systems are not members of galactic clusters or associations and being field stars 
would not be considered to be very young. This interpretation is in accord with the general tendency for the stars to lie above (or to the right of) the zero-age main sequence. In accordance with the prevailing view also is the fact that generally the later type components lie closer to the zero-age sequence than do the earlier.

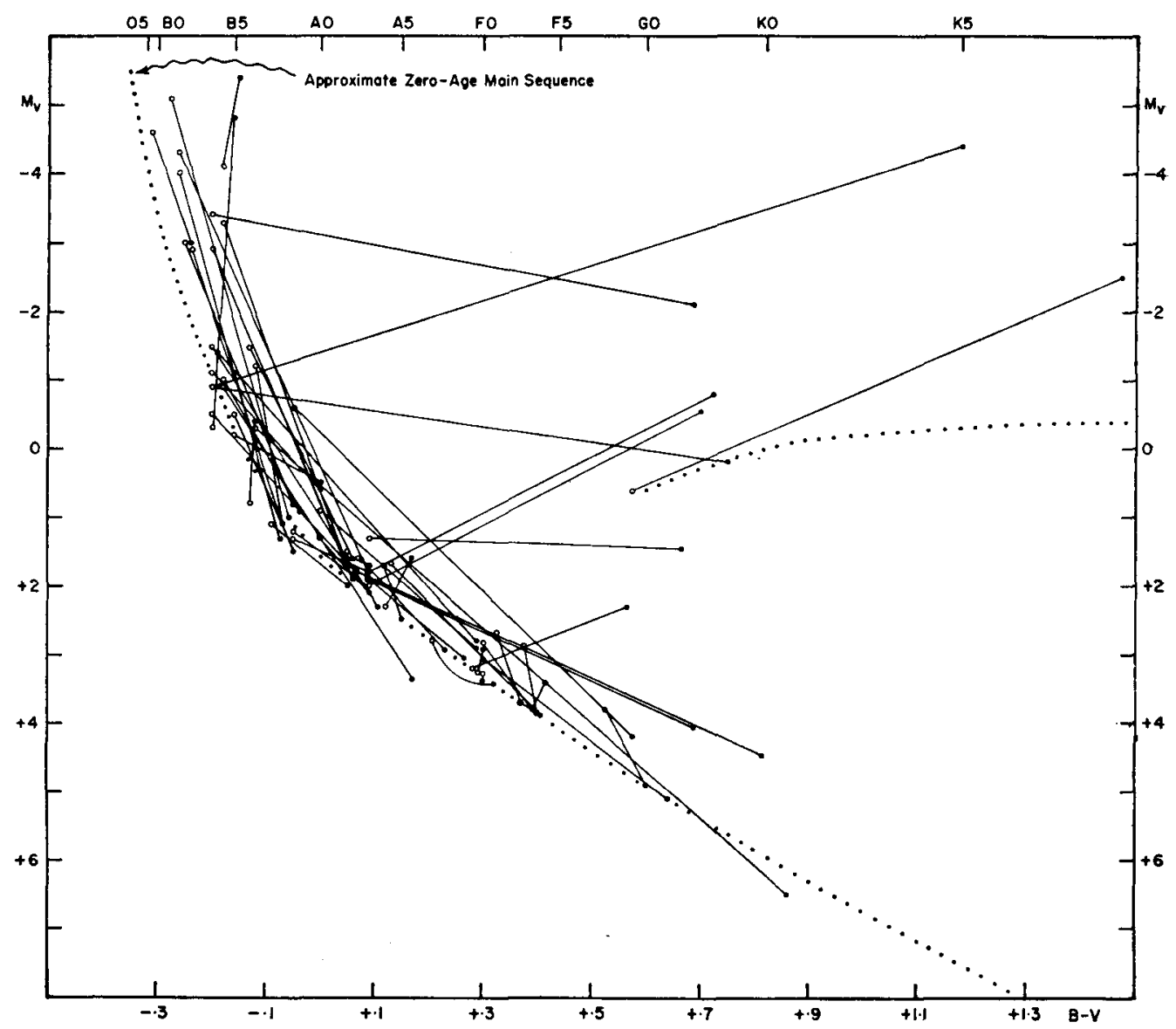

Fig. 3. Hertzsprung-Russell diagram for visual binaries.

Also in accord with current ideas of stellar evolution are the presence of 'classical' evolved systems where an early type main-sequence star is coupled with a solar type giant, e.g. $\epsilon$ Boo and . Can, and there is a more advanced stage represented by A.D.S.8489 where both components have moved a long way from the main sequence.

There are some interesting systems which appear not to agree with current ideas of the course of stellar evolution. Perhaps the most spectacular are systems such as A.D.S.2559, 1262, I0993 (95 Her) where similar magnitudes of the components are accompanied by large spectral type differences. It does not seem to be easy to explain these on the usual ideas of evolution: are these examples of a violation of the assumption that the equilibrium position of a star on the main sequence is dependent entirely on the mass of the original proto-star?

Another interesting group is that in which similar spectra are accompanied by substantial 
magnitude differences. Examples are 67 Oph (A.D.S. Iog66) and we might include $\epsilon$ Persei and $\eta$ Persei. The special feature of these systems is the enormous ratio of light of the components contrasted with apparently similar rates of evolution. The secondary stars should be evolving relatively so slowly that by the time they reach the main sequence the primary stars would be expected to be evolved far off it. Even if the secondaries have just 'arrived' should not the very luminous companions have long ago exhausted their hydrogen fuel supply and become red giants, or supergiants?

In summary one may perhaps conclude that visual binaries support, in most cases, current views of stellar evolutionary processes, but that there are some interesting nonconformists. It would seem that visual binaries are especially valuable in the study of stellar evolution because the relative position of the components on the colour-magnitude diagram can be ascertained without ambiguity and, ultimately some knowledge of the masses of the plotted stars may be obtained. This last unique advantage afforded by the visual binaries indicates that continued observations of binary systems will be of great significance in understanding evolutionary changes in stars.

\section{RADIAL VELOCITIES FROM HIGH DISPERSION SPECTROGRAMS}

The great power of modern coudé spectrographs appears to be particularly suited to investigations of visual binaries. The measurement of line intensities and the subsequent determination of the chemical composition of the atmospheres of visual binaries could furnish important information on the process of stellar evolution. Evolutionary changes in stars which are coeval, but in which the evolutionary rates differ might thus be detected. The most powerful spectroscopic apparatus will likely be needed in this problem.

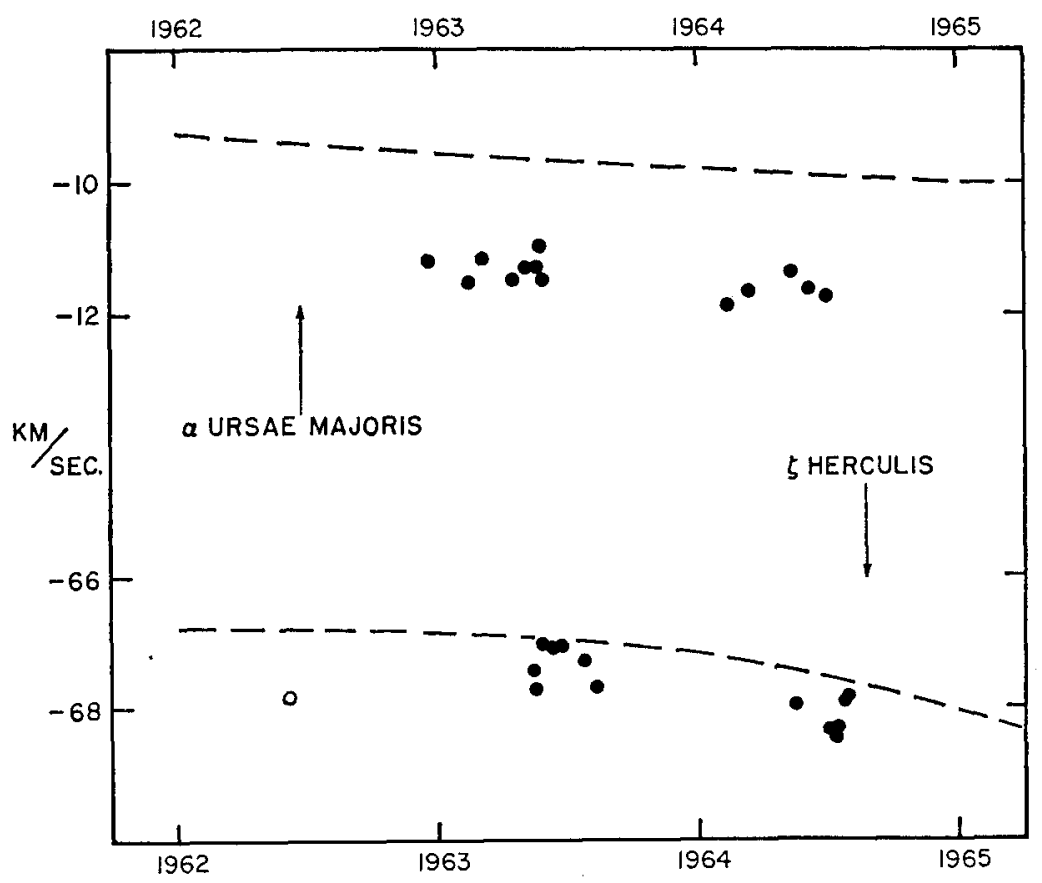

Fig. 4. Recent high-dispersion radial-velocity measures of $\alpha \mathrm{UMa}$ and $\zeta$ Her compared with the previously published velocity curves. 
The great accuracy with which radial velocities can be measured with coudé spectrographs finds an immediate and valuable application in supplementing astrometric orbital data and thus enabling us to calculate with high accuracy the masses and absolute magnitudes of the components of visual binaries. Radial velocities of solar type stars obtained with the coudé spectrograph at Victoria give a mean error of $\pm 0.2 \mathrm{~km} / \mathrm{sec}$ for one plate if suitable precautions are observed and this is the kind of accuracy required to study in detail the small orbital velocities encountered in visual binaries.

Fig. 4 shows a plot of some radial velocities of $\alpha \mathrm{UMa}$ and $\zeta \mathrm{Her}$ obtained recently at Victoria, compared with parts of the radial-velocity curves published some time ago. The curves require some adjustment to fit the velocities, but this may well be a simple systematic correction. Of more interest is the fact that a few observations give a velocity with great precision: for example four plates of $\alpha$ UMa give a mean velocity for May 1963 with a mean error of $\pm 0.12 \mathrm{~km} / \mathrm{sec}$. In addition to orbital determinations the high-dispersion velocities will make it possible to detect and to investigate irregularities in the motion and even, as suggested some years ago by Struve, to begin a direct search for planetary-like bodies by studying periodic distortions of orbital velocity-curves.

\section{Acknowledgments}

Thanks are due to all those members of the staff who participated in the observing of these stars. We are also grateful to Mr E. K. Lee, who made the spectrophotometric measures of $\mathrm{H} \gamma$ and to $\mathrm{Mr}$ F. Gutmann who helped in measuring the radial velocities.

\section{REFERENCES}

r. Luyten, W. J. Publ. astr. Obs. Univ. Minnesota, 3, no. 12, 1963.

2. McLaughlin, D. B. Publ. Obs. Univ. Michigan, 6, 3, 1934.

3. Struve, O., Huang, S.-S. Occ. Notes R. astr. Soc. no. 19, 1957.

4. Petrie, R. M. Publ. Dom. Astrophys. Obs. Victoria, 12, 3 17, 1964.

5. Muller, P. Ann. Obs. Strasbourg, 5, fasc. I, 2, 3, 4, I951; $\mathcal{~ F . ~ O b s e r v a t e u r s , ~ 3 5 , ~ 1 7 7 , ~ 1 9 5 2 ; ~}$ 40, $157,1957$.

6. Wallenquist, A. Ark. Mat. Astr. Fys., 30A, no. 8, $1944=$ Uppsala astr. Obs. Medd. no. 85; Ark. Mat. Astr. Fys., 31A, no. 16, 1944 =Uppsala astr. Obs. Medd. no. 87; Ark. Astr., 1, 193, I950 = Uppsala astr. Obs. Medd. no. 103; Ark. Astr., 2, 171, 1958 = Uppsala astr. Obs. Medd. no. I I 8.

7. Eggen, O. J. Astr. F., 68, 483, I963.

8. Tolbert, C. R. Astrophys. $\mathcal{F} .$, 139, $1105,1964$.

9. Arp, H. C. Handbuch der Physik, Springer Verlag, Berlin, 5I, 96, $195^{8}$.

ro. Johnson, H. L. Stars and Stellar Systems, University of Chicago Press, Chicago, 3, 214, $216,1963$.

II. Blaauw, A. Stars and Stellar Systems, University of Chicago Press, Chicago, 3, 407, 1963.

12. Keenan, P. C. Stars and Stellar Systems, University of Chicago Press, Chicago, 3, 92, 1963.

\section{DISCUSSION}

Underhill: The problem of systems like $\zeta$ Persei, $\epsilon$ Persei and 67 Ophiuchi in which the difference in spectral type is small but $\Delta M_{\mathrm{v}}$ is large arises, I think, because from the spectral type one assumes a bolometric correction. Theoretical and observational astrophysical studies of early-type atmospheres are giving me the feeling that the $B$ type supergiants do not necessarily have as large bolometric corrections as those of main-sequence stars of the same type. 
The spectrum is more or less a camouflage and is not necessarily a secure indicator of $T_{\text {eff }}$ and thus of the bolometric correction.

Petrie: The spectral types are estimated, but there are $B-V$ data and they are plotted on the HR diagram in $B-V$.

Underhill: The colour $(B-V)$ is not a more reliable indicator of $T_{\text {eff }}$ than the spectrum is. The difficulty is to relate the strength of the strong lines in a unique manner to $T_{\text {eff. }}$ My suspicions are that the relation is different in the case of luminous stars from the relation valid for main-sequence stars, and that for given relative line strengths, $T_{\text {eff }}$ is smaller in the luminous stars.

Popper: $\mathrm{H}_{\gamma}$ or $\mathrm{H}_{\beta}$ equivalent widths correlate well with luminosity with small scatter. $B-V$ correlates well with $M_{\mathrm{v}}$ in cluster $C-M$ diagrams. So perhaps these quantities depend on fundamental properties of the star, rather than on superficial effects as suggested by Miss Underhill.

Kraft: Regarding binaries with one member 6 magnitudes brighter than the companion and both near the main sequence, cannot the nuclear time-scale for the primary, and contraction time-scale for the secondary be made compatible by using the Hayashi tracks for the secondary?

Batten: The diagram is simply a plot of the observed positions of the stars in the HR diagram. Our idea was that the expected difference of evolutionary stages between the two components of such systems is very large on any reasonable time-scale, but this does not seem to be the case.

Schwarzschild: What worries me most in Dr Petrie's diagram are the two binaries with the primaries on the main sequence but the fainter components above the main sequence by about I magnitude, at absolute magnitude around +3 or +4 . Are these observations so sure that the fainter components cannot be pushed onto the main sequence?

Batten: One of these stars is one for which the evidence of physical connection is still weak. There is some evidence, however, and these systems remind one strongly of the much closer semi-detached systems with subgiant secondaries.

Schwarzschild: Are they wide pairs?

Batten: Yes, indeed.

\section{PHYSICAL DATA FROM NORMAL BINARIES}

\section{M. Popper}

\section{INTRODUCTION}

Starting with his pioneering studies of $U$ Cephei and $\beta$ Lyrae, Prof. Struve and a number of able collaborators, several of whom are here today, demonstrated that superficial effects are present in many close binaries that influence the spectrographic observations in such a way that the true orbital velocity variations, in particular, may not always be readily obtainable. Struve's principal interest was in studying these effects in order to understand their physical nature, their causes, and their possible influence on the evolutionary history of the system. Later speakers will emphasize these aspects of the topic. 